\title{
Immunomodulation as one of the key hallmarks of PUVA therapy: results from a mouse lymphoma skin model
}

Saptaswa Dey ${ }^{1}$, Pablo Vieyra-Garcia ${ }^{1}$, Theresa Benezeder ${ }^{1}$, Peter Wolf $^{1}$

${ }^{1}$ Research Unit for Photodermatology, Department of Dermatology, Medical University of Graz, Graz, Austria MOLIN

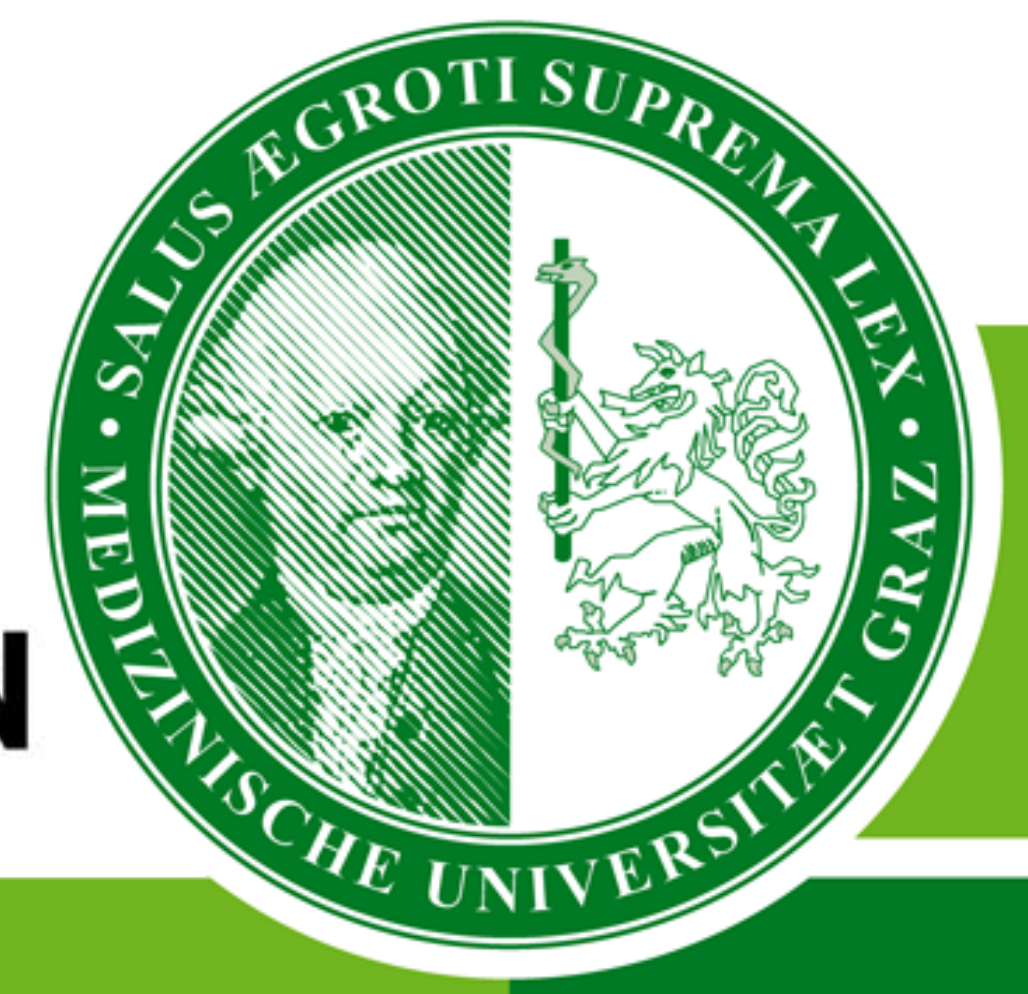

\section{Medical University of Graz}

\section{Background}

Cutaneous T-cell lymphoma (CTCL) represents heterogeneous group of neoplastic disorders characterized by chronic inflammation and primary accumulation of malignant $\mathrm{T}$ cells in the skin [1]. Lesional skin of CTCL patients is colonized by T-cells with aberrant phenotype and non-malignant immune cells, both possibly driven by soluble inflammatory mediators.

PUVA (Psoralen + UVA) is one of the gold standard of treatment for early stages of CTCL [2]. The combination of psoralen and UVA irradiation is frequently used to treat these patients with a success rate of more than $70 \%$ of complete clinical response.

DNA damage in malignant cells triggered by PUVA is thought to be the main mechanism of action of this therapy, however, the significance of PUVA-induced DNA and membrane damage-related immunomodulation and its potential contribution to clearance of CTCL lesions remains to be elucidated.

\section{Hypothesis \& Aims}

Immunomodulation is one of the key hallmark of PUVA mediated therapeutic effects in cutaneous $\mathrm{T}$ cell lymphoma

Aims: Mapping the pathways of PUVA mediated immunomodulation in CTCL

$\Rightarrow$ Analyzing T cell population

$\Rightarrow$ Analyzing transcription factors and mapping their role in PUVA mediated

\section{Establishment of murine lymphoma skin model}

To get a better understanding of how PUVA works we established a murine model of CTCL where we injected EL-4 cells subcutaneously on the back of a C57BL/6 mice and treated them with PUVA therapy.

Animals were sacrificed at various time points to collect tumor tissue and analyze lymphocytic distribution.

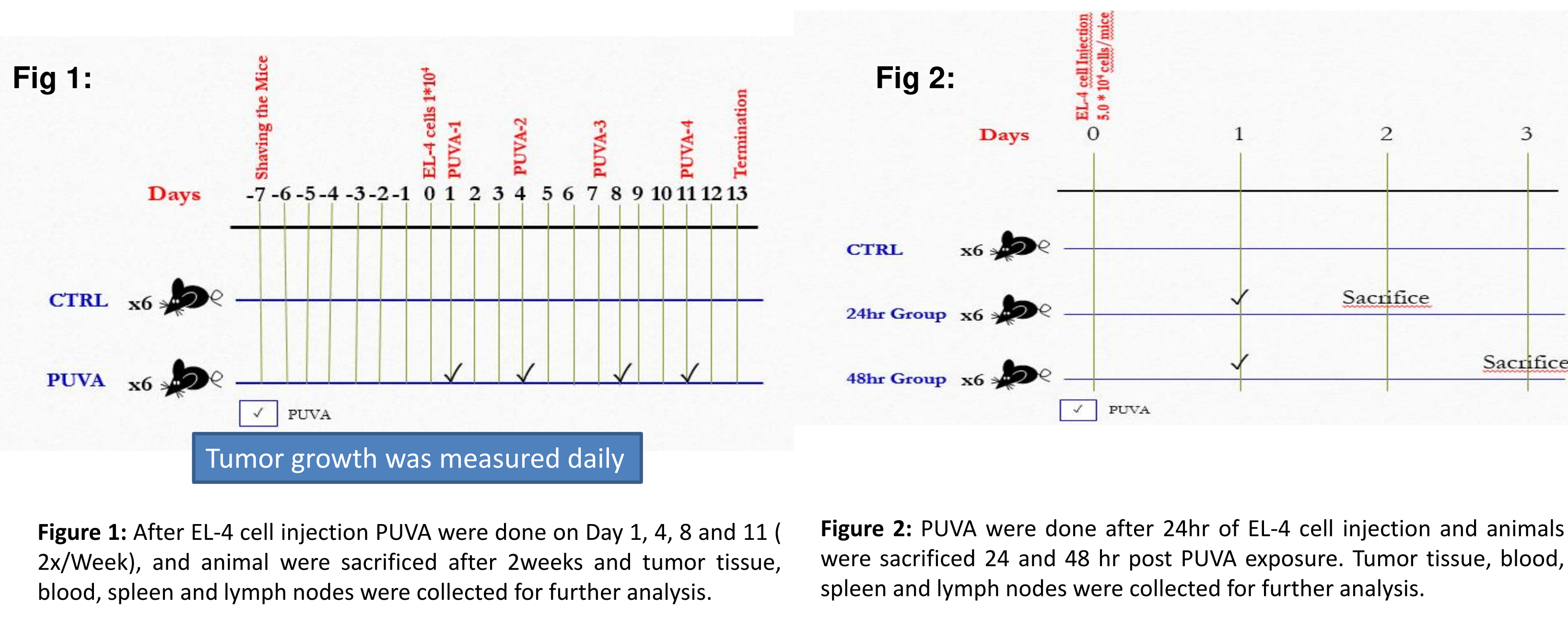

\section{Materials \& Methods}

PUVA dose: The mice were painted on their back with either $200 \mu$ l of 8-methoxypsoralen (8-MOP) in ethanol $(0.1 \mathrm{mg} / \mathrm{ml})$ or $200 \mu \mathrm{l}$ of vehicle (95\% ethanol). UVA irradiation was performed only on psoralen-painted mice after 15 min using a Waldmann Medizintechnik UV 236 A therapy lamp (Villingen-Schwenningen, Germany), filtered for the emission at $315-400 \mathrm{~nm}$ at a distance around $12 \mathrm{~cm}$ from the dorsal skin of the mouse. The UVA dose used was $1.5 \mathrm{~J} / \mathrm{cm}^{2}[3]$.

To study the systemic immunosuppressive effect of PUVA two separate experiments were performed. In one experiment (Figure 2) mice were sacrificed at 24 and $48 \mathrm{hr}$ post one exposure of PUVA treatment and in other experiment (Figure 1) the PUVA treatment was performed two times a week for two weeks.

$\Rightarrow$ Flow Cytometry were used to analyze lymphocytic distribution and immunomodulatory transcription factors in blood spleen and lymph nodes. Immunohistological staining was done to analyze collected tumor tissue. Statistical comparisons were done using student t-test (GraphPad Prism).

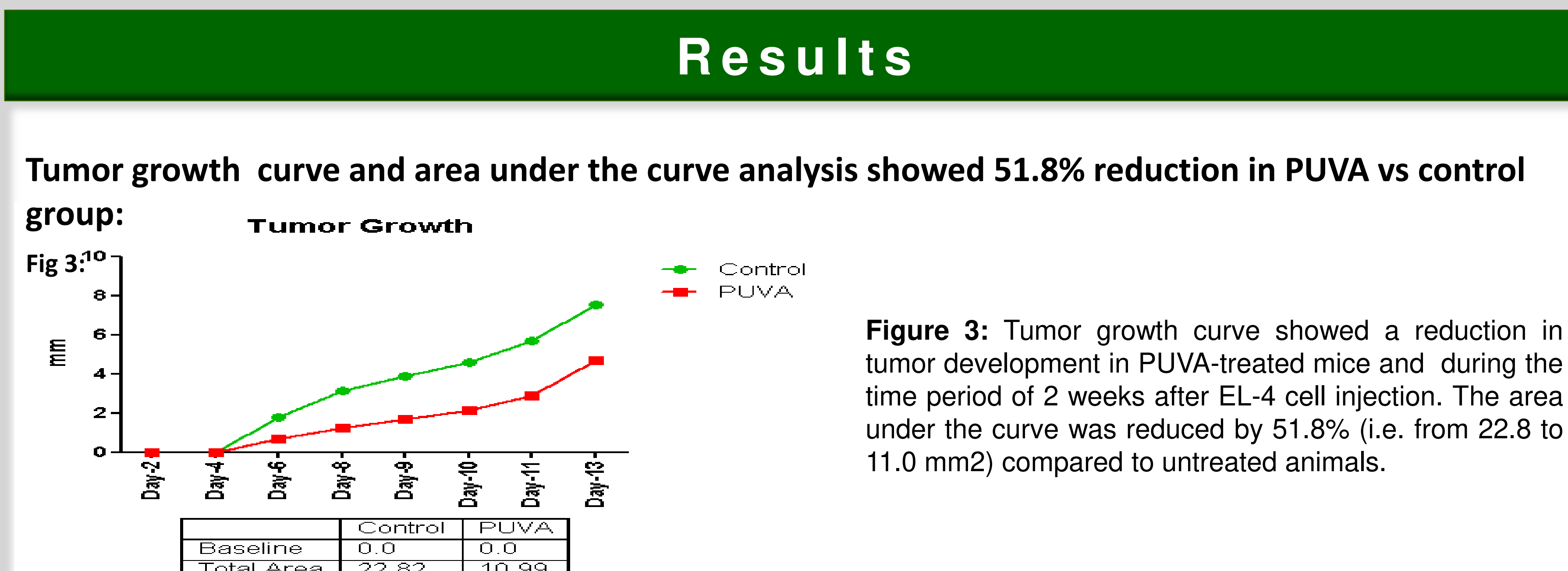

PUVA reduces CD4 \& CD8+ T-cells in lymph nodes, spleen and blood:

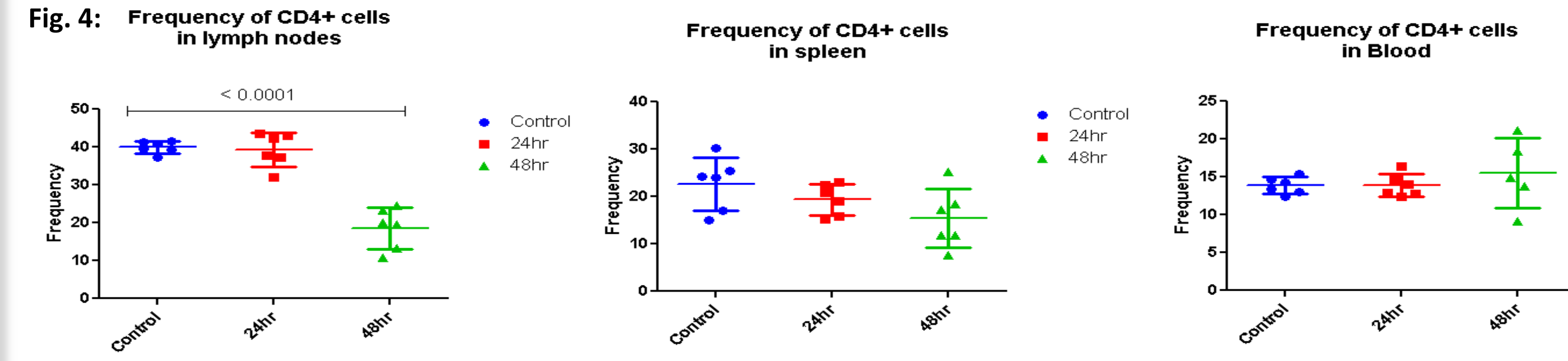

Figure 4: Analysis of the early effect of PUVA by flow cytometry showed that 48h after a single exposure to PUVA the tota percentage of CD4+ T-cells was reduced by $53.4 \%$ in lymph nodes but not significantly in spleen and blood.

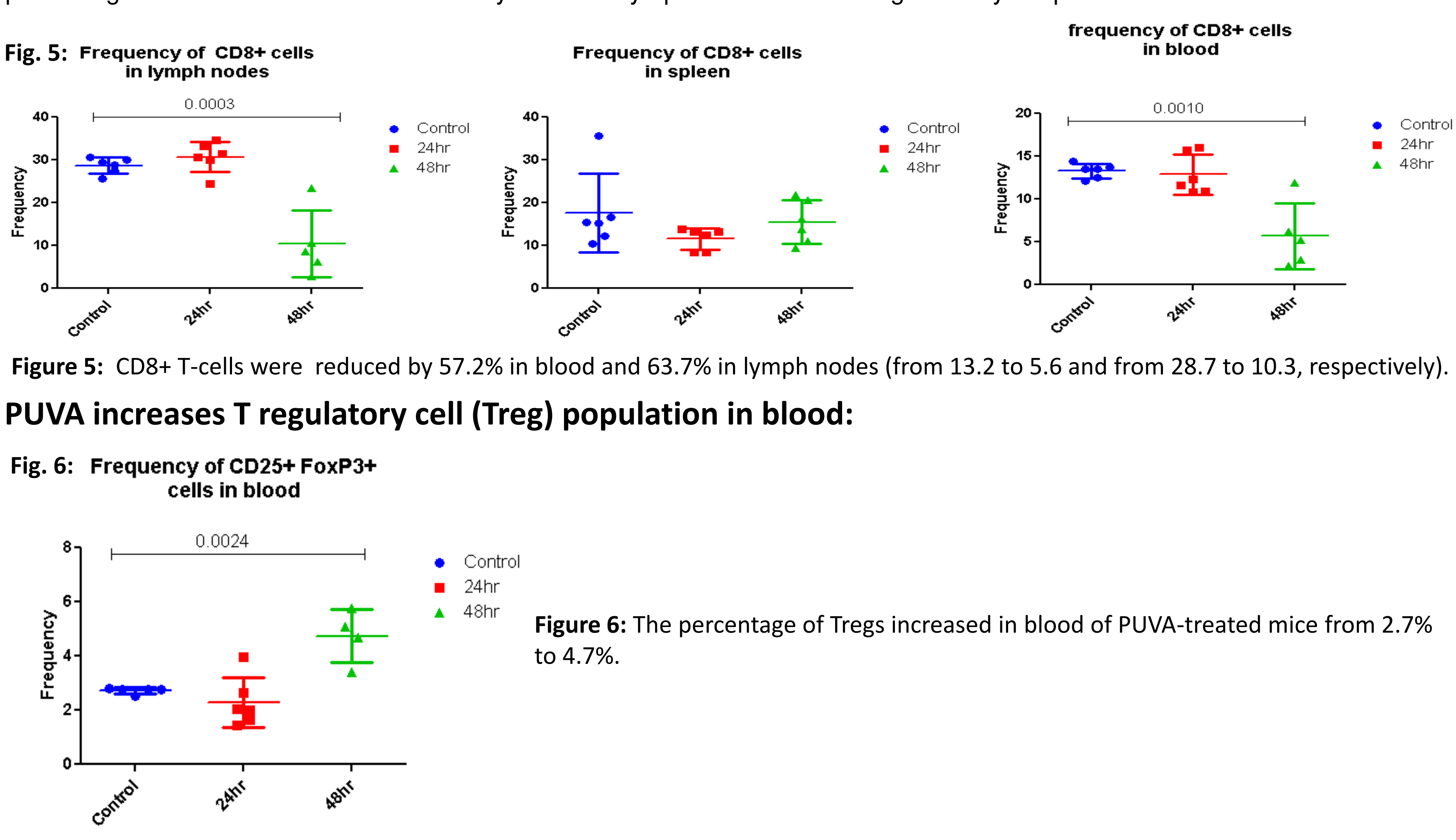

PUVA increses the expression of the MHC class II negative regulator LAG-3:

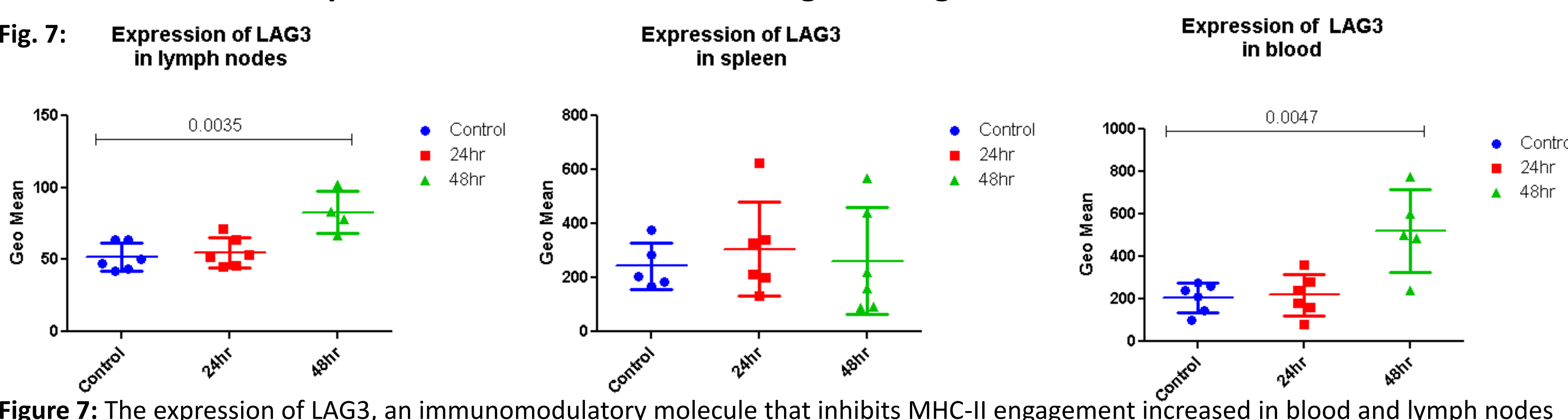
Figure 7: The expression of $L A G 3$, an immunomodity
(from 206 to 521 and 51.7 to 82.7 , respectively).

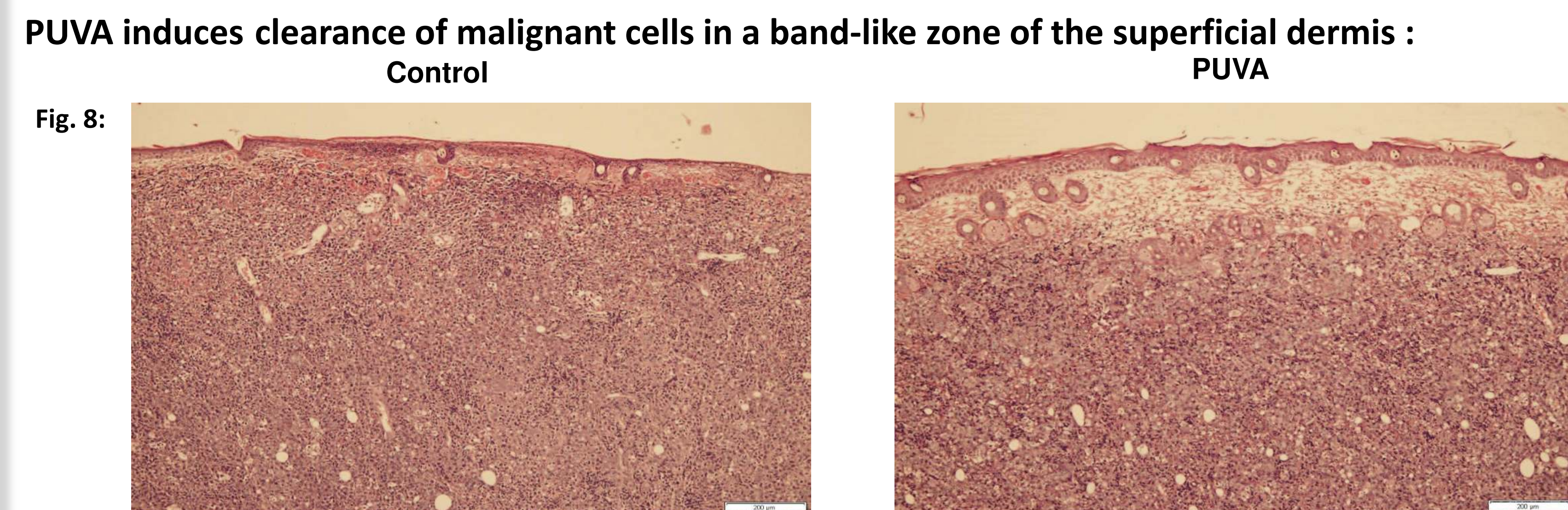
Figure 8: In tissue samples obtained 2 weeks after EL-4 cell injection, histological analysis of tumorous skin showed clearance
of malignant cells in a band-like zone of the superficial dermis adjacent to the epidermis and a decrease of overall epidermal thickness in PUVA-treated mice. Scale bars, $200 \mu \mathrm{m}$.

\section{Conclusion}

- Our mouse model recapitulates the hallmarks of the therapeutic effect of PUVA in CTCL by reducing tumor development and partially clearing the malignant cells from the tissue.

PUVA therapy triggered a reduction of systemic levels of CD4+ and CD8+ T-cells as well as an increase

The upregulation of LAG3 in PUVA-treated mice suggests an important role of this molecule in the immunomodulatory effect of this therapy.

Together these results highlight the involvement of immunomodulation in the therapeutic mechanisms of PUVA in CTCL by easing the tumor burden in lesional skin, possibly by affecting LAG3 and Tregs.

These results suggest that the immunomodulatory effects of PUVA on T cells and their role in therapeutic effect of CTCL.

\section{Acknowledgement}

We thank Gerlinde Mayer and Isabella Bambach for technical help.

This work was supported by Austrian Science Fund (FWF W1241). 